Bull. Korean Math. Soc. 50 (2013), No. 5, pp. 1555-1565

http://dx.doi.org/10.4134/BKMS.2013.50.5.1555

\title{
GENERALIZED DISCRETE HALANAY INEQUALITIES AND THE ASYMPTOTIC BEHAVIOR OF NONLINEAR DISCRETE SYSTEMS
}

\author{
LiguANG XU
}

\begin{abstract}
In this paper, some new generalized discrete Halanay inequalities are established. On the basis of these new established inequalities, we obtain the attracting set and the global asymptotic stability of the nonlinear discrete systems. Our results established here extend the main results in [R. P. Agarwal, Y. H. Kim, and S. K. Sen, New discrete Halanay inequalities: stability of difference equations, Commun. Appl. Anal. 12 (2008), no. 1, 83-90] and [S. Udpin and P. Niamsup, New discrete type inequalities and global stability of nonlinear difference equations, Appl. Math. Lett. 22 (2009), no. 6, 856-859].
\end{abstract}

\section{Introduction}

In 1966, Halanay proved the following famous result.

Halanay Inequality (Halanay [9, pp. 378-380]). If

$$
f^{\prime}(t) \leq-\alpha f(t)+\beta \sup _{[t-\tau, t]} f(s) \text { for } t \geq t_{0}
$$

and $\alpha>\beta>0$, then there exist $\gamma>0$ and $K>0$ such that

$$
f(t) \leq K e^{-\gamma\left(t-t_{0}\right)} \text { for } t \geq t_{0} .
$$

Since then, Halanay inequality has widely been applied to the stability analysis of delay differential systems (see e.g. $[3,5,7,8,9,11,12]$ ). At the same time, various generalized Halanay inequalities have been presented and used by many authors (see e.g. $[6,10,13,14,15,17,18,19,20,21,22,23,24,25])$. In particular, in $[1,16]$, the authors consider the following discrete Halanay-type inequalities in order to study some discretized versions of functional differential equations.

Received August 3, 2012

2010 Mathematics Subject Classification. 26D10, 26D20, 39A10, 39A70.

Key words and phrases. Halanay inequality, discrete systems, attracting set, global asymptotic stability.

The work is supported by National Natural Science Foundation of China under Grants 11101367 and 61074039. 
Theorem A (Udpin and Niamsup, [16, Theorem 2.1]). Let $q_{i} \in \mathbb{R}_{0}^{+}, h_{i} \in \mathbb{Z}^{+}$, $i=1, \ldots, r$, where $0=h_{0}<h_{1}<\cdots<h_{r}$ and $\sum_{i=0}^{r} q_{i}<p \leq 1$, and let $\left\{x_{j}\right\}_{j \in \mathbb{Z}^{-h_{r}}}$ be a sequence of real numbers satisfying the inequality

$$
\Delta x_{n} \leq-p x_{n}+\sum_{i=0}^{r} q_{i} x_{n-h_{i}}, n \in \mathbb{Z}^{0} .
$$

Then there exists $\lambda_{0} \in(0,1)$ such that

$$
x_{n} \leq \max \left\{0, x_{0}, x_{-1}, \ldots, x_{-h_{r}}\right\} \lambda_{0}^{n}, n \in \mathbb{Z}^{0} .
$$

Moreover, $\lambda_{0}$ may be chosen as the smallest root of the polynomial

$$
P(\lambda)=\lambda^{h_{r+1}}-\left(1-p+q_{0}\right) \lambda^{h_{r}}-q_{1} \lambda^{h_{r}-h_{1}}-\cdots-q_{r-1} \lambda^{h_{r}-h_{r-1}}-q_{r}
$$

which lies in the interval $(0,1)$.

Theorem B (Udpin and Niamsup, [16, Theorem 2.2]). Let $p, \alpha_{i}, \beta_{i} \in \mathbb{R}^{+}$, $h_{i} \in \mathbb{Z}^{+}, i=1, \ldots, r$, where $0=h_{0}<h_{1}<\cdots<h_{r}, \sum_{i=0}^{r} \alpha_{i}=1$ and $\Pi_{i=0}^{r} \beta_{i}<p \leq 1$. Let $\left\{x_{n}\right\}_{n \in \mathbb{Z}^{-h_{r}}}$ be a sequence of real numbers such that $x_{n-h_{i}}^{\alpha_{i}}$ are defined for all $i=1, \ldots, r ; n \in \mathbb{Z}^{0}$ which satisfies the inequality

$$
\Delta x_{n} \leq-p x_{n}+\left(\Pi_{i=0}^{r} \beta_{i}\right)\left(x_{n-h_{i}}^{\alpha_{i}}\right), n \in \mathbb{Z}^{0} .
$$

Then there exists $\lambda_{0} \in(0,1)$ such that

$$
x_{n} \leq \max \left\{0, x_{0}, x_{-1}, \ldots, x_{-h_{r}}\right\} \lambda_{0}^{n}, n \in \mathbb{Z}^{0} .
$$

Moreover, $\lambda_{0}$ can be chosen as the smallest root of the function

$$
F(\lambda)=\lambda-\left(\Pi_{i=0}^{r} \beta_{i}\right)\left(\lambda^{-\sum_{i=0}^{r} h_{i} \alpha_{i}}\right)+(p-1)
$$

which lies in the interval $(0,1)$.

Theorem C (Agarwal, Kim, and Sen, [1, Theorem 2.2]). Let $a_{i}, q_{i} \in \mathbb{R}_{0}^{+}, h_{i} \in$ $\mathbb{Z}^{0}, i=0, \ldots, r-1 ; a_{r}, q_{r} \in \mathbb{R}^{+}, h_{r} \in \mathbb{Z}^{+}$, where $0=h_{0}<h_{1}<\cdots<h_{r}$. Let $\alpha_{i}, \beta_{i} \in \mathbb{R}^{+}, \sum_{i=0}^{r} \alpha_{i}=1$ and $\left[(1-\delta) \Pi_{i=0}^{r} \beta_{i}+\delta \sum_{i=0}^{r} q_{i}\right]<\sum_{i=0}^{r} a_{i} \leq 1$, where $0 \leq \delta \leq 1$ is a constant. Also, let $\left\{x_{n}\right\}_{n \in \mathbb{Z}^{-h_{r}}}$ be a sequence of nonnegative real numbers satisfying the inequality

$$
\Delta x_{n} \leq \sum_{i=0}^{r}\left(\delta q_{i} x_{n-h_{i}}-a_{i} x_{n}\right)+(1-\delta)\left(\Pi_{i=0}^{r} \beta_{i}\right)\left(x_{n-h_{i}}^{\alpha_{i}}\right), n \in \mathbb{Z}^{0} .
$$

Then there exists a constant $\lambda_{0} \in(0,1)$ such that

$$
x_{n} \leq \max \left\{0, x_{0}, x_{-h_{1}}, \ldots, x_{-h_{r}}\right\} \lambda_{0}^{n}, n \in \mathbb{Z}^{0} .
$$

Moreover, $\lambda_{0}$ can be chosen as the root in the interval $(0,1)$ of the equation

$$
\lambda+\left(\sum_{i=0}^{r} a_{i}-1\right)-(1-\delta)\left(\Pi_{i=0}^{r} \beta_{i}\right)\left(\lambda^{-\sum_{i=0}^{r} \alpha_{i} h_{i}}\right)-\delta \sum_{i=0}^{r} q_{i} \lambda^{-h_{i}}=0 .
$$


Obviously, these discrete Halanay-type inequalities are important tools for investigating the stability of discrete systems. However, the equilibrium point sometimes does not exist in many real systems, especially in nonlinear dynamical systems. Therefore, an interesting subject is to discuss the attracting set of nonlinear discrete systems. However, the foregoing discrete Halanay-type inequalities are ineffective for studying the attracting sets of nonlinear discrete systems. With motivation from the above discussions, our main aim in the present paper is to improve the foregoing inequalities such that it is effective for studying the attracting sets of nonlinear discrete systems. We also illustrate the application of these inequalities.

\section{Generalized discrete Halanay inequalities}

Throughout this paper, unless otherwise specified, we use the following notations. Let $\mathbb{R}$ denote the set of all real numbers, $\mathbb{R}^{+}$the set of positive real numbers, $\mathbb{R}_{0}^{+}$the set of nonnegative real numbers, $\mathbb{Z}$ the set of integers, $\mathbb{Z}^{+}$the set of positive integers, and $\mathbb{Z}^{-r}=\{z \in \mathbb{Z}: z \geq-r\}$. For a sequence of real number $\left\{x_{n}\right\}$, the difference operator $\Delta$ on $x_{n}$ is defined as $\Delta x_{n}=x_{n+1}-x_{n}$.

In this section, we introduce some new generalized discrete Halanay inequalities which will be used to study the attracting set and the global asymptotic stability of the nonlinear discrete systems. We need the following lemma in the discussions of our main results.

Lemma 2.1 (Arithmetic-mean-geometric-mean inequality [4]). For $x_{i} \geq 0$, $\alpha_{i}>0$ and $\sum_{i=1}^{n} \alpha_{i}=1$,

$$
\prod_{i=1}^{n} x_{i}^{\alpha_{i}} \leq \sum_{i=1}^{n} \alpha_{i} x_{i}
$$

the sign of equality holds if and only if $x_{i}=x_{j}$ for all $i, j \in \mathcal{N}$.

Theorem 2.2. Let $a_{i}, q_{i}, \Upsilon \in \mathbb{R}_{0}^{+}, h_{i} \in \mathbb{Z}^{0}, i=0, \ldots, r-1 ; a_{r}, q_{r} \in \mathbb{R}^{+}$, $h_{r} \in \mathbb{Z}^{+}$, where $0=h_{0}<h_{1}<\cdots<h_{r}$. Let $\alpha_{i}, \beta_{i} \in \mathbb{R}^{+}, \sum_{i=0}^{r} \alpha_{i}=1$ and $\left[(1-\delta) \Pi_{i=0}^{r} \beta_{i}+\delta \sum_{i=0}^{r} q_{i}\right]<\sum_{i=0}^{r} a_{i} \leq 1$, where $0 \leq \delta \leq 1$ is a constant. Also let $\left\{x_{n}\right\}_{n \in \mathbb{Z}^{-h_{r}}}$ be a sequence of real numbers satisfying the inequality

$$
\Delta x_{n} \leq \sum_{i=0}^{r}\left(\delta q_{i} x_{n-h_{i}}-a_{i} x_{n}\right)+(1-\delta)\left(\Pi_{i=0}^{r} \beta_{i}\right)\left(\sum_{i=0}^{r} \alpha_{i} x_{n-h_{i}}\right)+\Upsilon, n \in \mathbb{Z}^{0} .
$$

Then there exists $\lambda_{0} \in(0,1)$ such that

$$
x_{n} \leq \max \left\{0, x_{0}, x_{-h_{1}}, \ldots, x_{-h_{r}}\right\} \lambda_{0}^{n}+\Lambda, n \in \mathbb{Z}^{0},
$$

where $\Lambda=\left(\sum_{i=0}^{r} a_{i}-\delta \sum_{i=0}^{r} q_{i}-(1-\delta) \Pi_{i=0}^{r} \beta_{i}\right)^{-1} \Upsilon$. Moreover, $\lambda_{0}$ can be chosen as the root in the interval $(0,1)$ of the equation

$$
\lambda+\left(\sum_{i=0}^{r} a_{i}-1\right)-(1-\delta)\left(\Pi_{i=0}^{r} \beta_{i}\right)\left(\sum_{i=0}^{r} \alpha_{i} \lambda^{-h_{i}}\right)-\delta \sum_{i=0}^{r} q_{i} \lambda^{-h_{i}}=0 .
$$


Proof. Let $y_{n}$ be a solution of the difference equation

$$
\Delta y_{n}=\sum_{i=0}^{r}\left(\delta q_{i} y_{n-h_{i}}-a_{i} y_{n}\right)+(1-\delta)\left(\Pi_{i=0}^{r} \beta_{i}\right)\left(\sum_{i=0}^{r} \alpha_{i} y_{n-h_{i}}\right)+\Upsilon, n \in \mathbb{Z}^{0} .
$$

Since $\left(1-\sum_{i=0}^{r} a_{i}\right) \geq 0, q_{i} \in \mathbb{R}_{0}^{+}, \beta_{i} \in \mathbb{R}^{+}$, it is easy to prove that if $x_{n}$ satisfies (2.1) and $x_{n} \leq y_{n}$ for $n=-h_{r}, \ldots, 0$, then $x_{n} \leq y_{n}$ for all $n \in \mathbb{Z}^{0}$. For a given $K>0$ and $\lambda \in(0,1)$, the sequence $\left\{y_{n}\right\}$ defined by $y_{n}=K \lambda_{n}+\Lambda$ is a solution of (2.4) if and only if $\lambda$ is a root of the polynomial (2.3). In fact,

$$
\begin{aligned}
& y_{n}=K \lambda^{n}+\Lambda \text { is a solution of (2.4) } \\
& \Longleftrightarrow K \lambda^{n+1}-K \lambda^{n}=\sum_{i=0}^{r}\left[\delta q_{i}\left(K \lambda^{n-h_{i}}+\Lambda\right)-a_{i}\left(K \lambda^{n}+\Lambda\right)\right] \\
& +(1-\delta)\left(\Pi_{i=0}^{r} \beta_{i}\right)\left(\sum_{i=0}^{r} \alpha_{i}\left(K \lambda^{n-h_{i}}+\Lambda\right)\right)+\Upsilon \\
& \Longleftrightarrow K \lambda^{n+1}-K \lambda^{n}\left(\sum_{i=0}^{r} a_{i}-1\right)-(1-\delta)\left(\Pi_{i=0}^{r} \beta_{i}\right)\left(\sum_{i=0}^{r} \alpha_{i} K \lambda^{n-h_{i}}\right) \\
& -\sum_{i=0}^{r} \delta q_{i}\left(K \lambda^{n-h_{i}}\right)+\left(\sum_{i=0}^{r} a_{i}-\delta \sum_{i=0}^{r} q_{i}-(1-\delta) \Pi_{i=0}^{r} \beta_{i}\right) \Lambda-\Upsilon=0 \\
& \Longleftrightarrow K \lambda^{n+1}-K \lambda^{n}\left(\sum_{i=0}^{r} a_{i}-1\right)-K \lambda^{n}(1-\delta)\left(\Pi_{i=0}^{r} \beta_{i}\right)\left(\sum_{i=0}^{r} \alpha_{i} \lambda^{-h_{i}}\right) \\
& -K \lambda^{n} \sum_{i=0}^{r} \delta q_{i}\left(\lambda^{-h_{i}}\right)=0 \\
& \Longleftrightarrow \lambda+\left(\sum_{i=0}^{r} a_{i}-1\right)-(1-\delta)\left(\Pi_{i=0}^{r} \beta_{i}\right)\left(\sum_{i=0}^{r} \alpha_{i} \lambda^{-h_{i}}\right)-\delta \sum_{i=0}^{r} q_{i} \lambda^{-h_{i}}=0
\end{aligned}
$$

$\Longleftrightarrow \lambda$ is a root of the polynomial (2.3).

Define a function by $F$ by

$$
F(\lambda)=\lambda+\left(\sum_{i=0}^{r} a_{i}-1\right)-(1-\delta)\left(\Pi_{i=0}^{r} \beta_{i}\right)\left(\sum_{i=0}^{r} \alpha_{i} \lambda^{-h_{i}}\right)-\delta \sum_{i=0}^{r} q_{i} \lambda^{-h_{i}}
$$

Since

$$
\begin{aligned}
\lim _{\lambda \rightarrow 0^{+}} F(\lambda)= & \left(\sum_{i=0}^{r} a_{i}-1\right)-(1-\delta)\left(\Pi_{i=0}^{r} \beta_{i}\right)\left(\sum_{i=0}^{r} \alpha_{i} \lim _{\lambda \rightarrow 0^{+}} \lambda^{-h_{i}}\right) \\
& -\delta \sum_{i=0}^{r} q_{i} \lim _{\lambda \rightarrow 0^{+}} \lambda^{-h_{i}}<0
\end{aligned}
$$


and $F(1)=\sum_{i=0}^{r} a_{i}-(1-\delta)\left(\Pi_{i=0}^{r} \beta_{i}\right)-\delta \sum_{i=0}^{r} q_{i}>0$, it follows from continuity of $F$ that there exists a real number $\lambda_{0} \in(0,1)$ such that $F\left(\lambda_{0}\right)=0$. Thus for any $K \in \mathbb{R}_{0}^{+}$, the sequence $K \lambda_{0}^{n}+\Lambda$ is a solution of (2.4). Let $K_{0}=$ $\max \left\{0, x_{0}, x_{-1}, \ldots, x_{-h_{r}}\right\}$. Then, $\left\{y_{n}\right\}=K_{0} \lambda_{0}^{n}+\Lambda$ is a solution of (2.4) and obviously we have $x_{n} \leq y_{n}$ for $n=-h_{r}, \ldots, 0$. Therefore, by using the first part of the proof, we conclude that $x_{n} \leq y_{n}=K_{0} \lambda_{0}^{n}+\Lambda, n \in \mathbb{Z}^{0}$.

Remark 2.3. When we take $\delta=1$ and let $p=\sum_{i=0}^{r} a_{i}$. By Theorem 2.2 we have the following result.

Theorem 2.4. Let $q_{i}, \Upsilon \in \mathbb{R}_{0}^{+}, h_{i} \in \mathbb{Z}^{+}, i=1, \ldots, r$, where $0=h_{0}<$ $h_{1}<\cdots<h_{r}$ and $\sum_{i=0}^{r} q_{i}<p \leq 1$, and let $\left\{x_{n}\right\}_{n \in \mathbb{Z}^{-h_{r}}}$ be a sequence of real numbers satisfying the inequality

$$
\Delta x_{n} \leq-p x_{n}+\sum_{i=0}^{r} q_{i} x_{n-h_{i}}+\Upsilon, n \in \mathbb{Z}^{0}
$$

Then there exists $\lambda_{0} \in(0,1)$ such that

$$
x_{n} \leq \max \left\{0, x_{0}, x_{-h_{1}}, \ldots, x_{-h_{r}}\right\} \lambda_{0}^{n}+\Lambda, n \in \mathbb{Z}^{0},
$$

where $\Lambda=\left(p-\sum_{i=0}^{r} q_{i}\right)^{-1} \Upsilon$. Moreover, $\lambda_{0}$ may be chosen as the smallest root of the polynomial

$$
P(\lambda)=\lambda^{h_{r+1}}-\left(1-p+q_{0}\right) \lambda^{h_{r}}-q_{1} \lambda^{h_{r}-h_{1}}-\cdots-q_{r-1} \lambda^{h_{r}-h_{r-1}}-q_{r}
$$

which lies in the interval $(0,1)$.

Remark 2.5. Suppose that $\Upsilon=0$ in Theorem 2.4. Then we get Theorem A (Udpin and Niamsup, [16, Theorem 2.1]).

Remark 2.6. By Lemma 2.1 and Theorem 2.2, we can obtain the following theorem.

Theorem 2.7. Let $a_{i}, q_{i}, \Upsilon \in \mathbb{R}_{0}^{+}, h_{i} \in \mathbb{Z}^{0}, i=0, \ldots, r-1 ; a_{r}, q_{r} \in \mathbb{R}^{+}$, $h_{r} \in \mathbb{Z}^{+}$, where $0=h_{0}<h_{1}<\cdots<h_{r}$. Let $\alpha_{i}, \beta_{i} \in \mathbb{R}^{+}, \sum_{i=0}^{r} \alpha_{i}=1$ and $\left[(1-\delta) \Pi_{i=0}^{r} \beta_{i}+\delta \sum_{i=0}^{r} q_{i}\right]<\sum_{i=0}^{r} a_{i} \leq 1$, where $0 \leq \delta \leq 1$ is a constant. Also let $\left\{x_{n}\right\}_{n \in \mathbb{Z}^{-h_{r}}}$ be a sequence of nonnegative real numbers satisfying the inequality

$$
\Delta x_{n} \leq \sum_{i=0}^{r}\left(\delta q_{i} x_{n-h_{i}}-a_{i} x_{n}\right)+(1-\delta)\left(\Pi_{i=0}^{r} \beta_{i}\right)\left(x_{n-h_{i}}^{\alpha_{i}}\right)+\Upsilon, n \in \mathbb{Z}^{0}
$$

Then there exists $\lambda_{0} \in(0,1)$ such that

$$
x_{n} \leq \max \left\{0, x_{0}, x_{-h_{1}}, \ldots, x_{-h_{r}}\right\} \lambda_{0}^{n}+\Lambda, n \in \mathbb{Z}^{0},
$$

where $\Lambda=\left(\sum_{i=0}^{r} a_{i}-\delta \sum_{i=0}^{r} q_{i}-(1-\delta) \Pi_{i=0}^{r} \beta_{i}\right)^{-1} \Upsilon$. Moreover, $\lambda_{0}$ can be chosen as the root in the interval $(0,1)$ of the equation

$$
\lambda+\left(\sum_{i=0}^{r} a_{i}-1\right)-(1-\delta)\left(\Pi_{i=0}^{r} \beta_{i}\right)\left(\sum_{i=0}^{r} \alpha_{i} \lambda^{-h_{i}}\right)-\delta \sum_{i=0}^{r} q_{i} \lambda^{-h_{i}}=0 .
$$


Proof. By (2.9) and Lemma 2.1, we get (2.1). Then, all the conditions of Theorem 2.2 are satisfied. By Theorem 2.2, we can obtain Theorem 2.7.

Remark 2.8. Suppose that $\Upsilon=0$ in Theorem 2.7. Then we get the main result of Theorem C (Agarwal, Kim and Sen, [1]).

Remark 2.9. When we take $\delta=0$. By Theorem 2.7 we have the following result.

Theorem 2.10. Let $a_{i}, \Upsilon \in \mathbb{R}_{0}^{+}, h_{i} \in \mathbb{Z}^{0}, i=0, \ldots, r-1 ; a_{r} \in \mathbb{R}^{+}, h_{r} \in \mathbb{Z}^{+}$, where $0=h_{0}<h_{1}<\cdots<h_{r}$. Let $\alpha_{i}, \beta_{i} \in \mathbb{R}^{+}, \sum_{i=0}^{r} \alpha_{i}=1$ and $\Pi_{i=0}^{r} \beta_{i}<$ $\sum_{i=0}^{r} a_{i} \leq 1$. Also let $\left\{x_{n}\right\}_{n \in \mathbb{Z}^{-h_{r}}}$ be a sequence of nonnegative real numbers satisfying the inequality

$$
\Delta x_{n} \leq-\sum_{i=0}^{r} a_{i} x_{n}+\left(\Pi_{i=0}^{r} \beta_{i}\right)\left(x_{n-h_{i}}^{\alpha_{i}}\right)+\Upsilon, n \in \mathbb{Z}^{0} .
$$

Then there exists $\lambda_{0} \in(0,1)$ such that

$$
x_{n} \leq \max \left\{0, x_{0}, x_{-h-1}, \ldots, x_{-h_{r}}\right\} \lambda_{0}^{n}+\Lambda, n \in \mathbb{Z}^{0},
$$

where $\Lambda=\left(\sum_{i=0}^{r} a_{i}-\Pi_{i=0}^{r} \beta_{i}\right)^{-1} \Upsilon$. Moreover, $\lambda_{0}$ can be chosen as the root in the interval $(0,1)$ of the equation

$$
\lambda+\left(\sum_{i=0}^{r} a_{i}-1\right)-\left(\Pi_{i=0}^{r} \beta_{i}\right)\left(\sum_{i=0}^{r} \alpha_{i} \lambda^{-h_{i}}\right)=0 .
$$

Remark 2.11. Suppose that $\Upsilon=0$ in Theorem 2.10, then we get the main result of Theorem B (Udpin and Niamsup, [16, Theorem 2.2]).

\section{Asymptotic behavior of discrete systems}

The inequalities obtained in Section 2 can be widely applied to research the asymptotic behavior of delay discrete dynamic systems. To illustrate the validity, consider the following discrete dynamic systems.

$$
\Delta x_{n}=-p x_{n}+f\left(n, x_{n}, x_{n-h_{1}}, \ldots, x_{n-h_{r}}\right),
$$

where $n, h_{i} \in \mathbb{Z}^{+}, i=1, \ldots, r \in \mathbb{Z}^{+}, p>0$. For any initial string $\left\{x_{-r}, x_{-r+1}\right.$, $\left.\ldots, x_{0}\right\},(3.1)$ has a unique solution which can be explicitly calculated $[1,16]$. However, it is difficult to obtain the attracting set and the global asymptotic stability using that form of solution. The following results give the attracting set and the global asymptotic stability of (3.1) by using the inequalities derived in Section 2.

Definition 3.1. The set $S \subset \mathbb{R}$ is called a global attracting set of (3.1), if for any initial string $\left\{x_{-r}, x_{-r+1}, \ldots, x_{0}\right\}$, the solution $\left\{x_{n}\right\}$ satisfies

$$
\operatorname{dist}\left(x_{n}, S\right) \rightarrow 0 \text { as } n \rightarrow \infty,
$$

where $\operatorname{dist}(\phi, S)=\inf _{\psi \in S} \rho(\phi, \psi)$ for $\phi \in \mathbb{R}, \rho(\cdot, \cdot)$ is any distance in $\mathbb{R}$. 
Theorem 3.2. Assume that there exist $q_{i}, \Upsilon \in \mathbb{R}_{0}^{+}, h_{i} \in \mathbb{Z}^{+}, q_{r} \in \mathbb{R}^{+}$, where $\sum_{i=0}^{r} q_{i}<p \leq 1$ such that

$$
\left|f\left(n, x_{n}, x_{n-h_{1}}, \ldots, x_{n-h_{r}}\right)\right| \leq \sum_{i=0}^{r} q_{i}\left|x_{n-h_{i}}\right|+\Upsilon
$$

for all $\left(n, x_{n}, x_{n-h_{1}}, \ldots, x_{n-h_{r}}\right) \in \mathbb{Z}^{0} \times \mathbb{R}^{r+1}$. Then, there exists $\lambda_{0} \in(0,1)$ such that every solution $\left\{x_{n}\right\}$ of (3.1) satisfies

$$
\left|x_{n}\right| \leq\left(\max _{-h_{r} \leq i \leq 0}\left\{\left|x_{i}\right|\right\}\right) \lambda_{0}^{n}+\left(p-\sum_{i=0}^{r} q_{i}\right)^{-1} \Upsilon, n \in \mathbb{Z}^{0},
$$

where $\lambda_{0}$ is chosen as in Theorem 2.4. As a consequence,

$$
S=\left\{\phi \in \mathbb{R}|| \phi \mid \leq\left(p-\sum_{i=0}^{r} q_{i}\right)^{-1} \Upsilon\right\}
$$

is a positive attracting set of (3.1).

Proof. As in [2], it is straightforward to show that every solution $\left\{x_{n}\right\}$ of (3.1) can be written in the form

$$
x_{n}=x_{0}(1-p)^{n}+\sum_{i=0}^{n-1}(1-p)^{n-i-1} f\left(i, x_{i}, x_{i-h_{1}}, \ldots, x_{i-h_{r}}\right), n \in \mathbb{Z}^{0} .
$$

By using (3.2), we obtain

$$
\left|x_{n}\right| \leq\left|x_{0}\right|(1-p)^{n}+\sum_{i=0}^{n-1}(1-p)^{n-i-1}\left(\sum_{j=0}^{r} q_{j}\left|x_{i-h_{j}}\right|+\Upsilon\right), n \in \mathbb{Z}^{0} .
$$

For each $n=-h_{r}, \ldots, 0$, let $v_{n}=\left|x_{n}\right|$ and for each $n \in \mathbb{Z}^{+}$, we let

$$
v_{n}=\left|x_{0}\right|(1-p)^{n}+\sum_{i=0}^{n-1}(1-p)^{n-i-1}\left(\sum_{j=0}^{r} q_{j}\left|x_{i-h_{j}}\right|+\Upsilon\right) .
$$

Then, we have $\left|x_{n}\right| \leq v_{n}, n \in \mathbb{Z}^{-h_{r}}$, and hence,

$$
\Delta v_{n}=-p v_{n}+\sum_{i=0}^{r} q_{i}\left|x_{n-h_{i}}\right|+\Upsilon \leq-p v_{n}+\sum_{i=0}^{r} q_{i} v_{n-h_{i}}+\Upsilon, n \in \mathbb{Z}^{0} .
$$

Therefore, by Theorem 2.4, we obtain

$$
\left|x_{n}\right| \leq v_{n} \leq\left(\max _{-h_{r} \leq i \leq 0}\left\{v_{i}\right\}\right) \lambda_{0}^{n}+\Lambda=\left(\max _{-h_{r} \leq i \leq 0}\left\{\left|x_{i}\right|\right\}\right) \lambda_{0}^{n}+\Lambda, n \in \mathbb{Z}^{0},
$$

where $\Lambda=\left(p-\sum_{i=0}^{r} q_{i}\right)^{-1} \Upsilon$, and $\lambda_{0}$ is chosen as in Theorem 2.4. This completes the proof of the theorem.

Remark 3.3. Suppose that $\Upsilon=0$ in Theorem 3.2. Then we get the following corollary. 
Corollary 3.4 (Udpin and Niamsup [16, Theorem 3.1]). Assume that there exist $q_{i} \in \mathbb{R}_{0}^{+}, h_{i} \in \mathbb{Z}^{+}, q_{r} \in \mathbb{R}^{+}$, where $\sum_{i=0}^{r} q_{i}<p \leq 1$ such that

$$
\left|f\left(n, x_{n}, x_{n-h_{1}}, \ldots, x_{n-h_{r}}\right)\right| \leq \sum_{i=0}^{r} q_{i}\left|x_{n-h_{i}}\right|
$$

for all $\left(n, x_{n}, x_{n-h_{1}}, \ldots, x_{n-h_{r}}\right) \in \mathbb{Z}^{0} \times \mathbb{R}^{r+1}$. Then, there exists $\lambda_{0} \in(0,1)$ such that every solution $\left\{x_{n}\right\}$ of (3.1) satisfies

$$
\left|x_{n}\right| \leq\left(\max _{-h_{r} \leq i \leq 0}\left\{\left|x_{i}\right|\right\}\right) \lambda_{0}^{n}, n \in \mathbb{Z}^{0},
$$

where $\lambda_{0}$ is chosen as in Theorem 2.4.

Theorem 3.5. Assume that $0<p \leq 1$. Let $q_{i}, \Upsilon \in \mathbb{R}_{0}^{+}, h_{i} \in \mathbb{Z}^{0}, i=$ $0, \ldots, r-1 ; q_{r} \in \mathbb{R}^{+}, h_{r} \in \mathbb{Z}^{+}$, where $0=h_{0}<h_{1}<\cdots<h_{r}$. Let $\alpha_{i}, \beta_{i} \in \mathbb{R}^{+}$, $\sum_{i=0}^{r} \alpha_{i}=1$ and $\left[(1-\delta) \Pi_{i=0}^{r} \beta_{i}+\delta \sum_{i=0}^{r} q_{i}\right]<p \leq 1$, where $0 \leq \delta \leq 1$ is a constant. If

$$
\left|f\left(n, x_{n}, x_{n-h_{1}}, \ldots, x_{n-h_{r}}\right)\right| \leq \sum_{i=0}^{r} \delta q_{i}\left|x_{n-h_{i}}\right|+(1-\delta)\left(\Pi_{i=0}^{r} \beta_{i}\right)\left|x_{n-h_{i}}\right|^{\alpha_{i}}+\Upsilon
$$

for all $\left(n, x_{n}, x_{n-h_{1}}, \ldots, x_{n-h_{r}}\right) \in \mathbb{Z}^{0} \times \mathbb{R}^{r+1}$, then there exists $\lambda_{0} \in(0,1)$ such that, for every solution $\left\{x_{n}\right\}$ of equation (3.1),

$$
\left|x_{n}\right| \leq\left(\max _{-h_{r} \leq i \leq 0}\left\{\left|x_{i}\right|\right\}\right) \lambda_{0}^{n}+\Lambda, n \in \mathbb{Z}^{0},
$$

where $\Lambda=\left(p-\delta \sum_{i=0}^{r} q_{i}-(1-\delta) \Pi_{i=0}^{r} \beta_{i}\right)^{-1} \Upsilon$, and $\lambda_{0}$ is chosen as in Theorem 2.7. As a consequence, $S=\{\phi \in \mathbb{R}|| \phi \mid \leq \Lambda\}$ is a positive attracting set of (3.1).

Proof. As in [2], it is straightforward to show that every solution $x_{n}$ of (3.1) can be written in the form

(3.13) $x_{n}=x_{0}(1-p)^{n}+\sum_{i=0}^{n-1}(1-p)^{n-i-1} f\left(i, x_{i}, x_{i-h_{1}}, \ldots, x_{i-h_{r}}\right), n \in \mathbb{Z}^{0}$.

By using (3.11), we obtain

$$
\begin{aligned}
\left|x_{n}\right| \leq & \left|x_{0}\right|(1-p)^{n} \\
& +\sum_{i=0}^{n-1}(1-p)^{n-i-1}\left(\sum_{j=0}^{r} \delta q_{j}\left|x_{i-h_{j}}\right|+(1-\delta)\left(\Pi_{j=0}^{r} \beta_{j}\right)\left|x_{i-h_{j}}\right|^{\alpha_{i}}+\Upsilon\right), n \in \mathbb{Z}^{0} .
\end{aligned}
$$

For each $n=-h_{r}, \ldots, 0$, let $v_{n}=\left|x_{n}\right|$ and for each $n \in \mathbb{Z}^{+}$, we let

$$
v_{n}=\left|x_{0}\right|(1-p)^{n}
$$




$$
+\sum_{i=0}^{n-1}(1-p)^{n-i-1}\left(\sum_{j=0}^{r} \delta q_{j}\left|x_{i-h_{j}}\right|+(1-\delta)\left(\Pi_{j=0}^{r} \beta_{j}\right)\left|x_{i-h_{j}}\right|^{\alpha_{i}}+\Upsilon\right) .
$$

Then, we have $\left|x_{n}\right| \leq v_{n}, n \in \mathbb{Z}^{-h_{r}}$, and hence,

$$
\begin{aligned}
\Delta v_{n} & =-p v_{n}+\sum_{i=0}^{r} \delta q_{i}\left|x_{n-h_{i}}\right|+(1-\delta)\left(\Pi_{i=0}^{r} \beta_{i}\right)\left|x_{n-h_{i}}\right|^{\alpha_{i}}+\Upsilon \\
& \leq-p v_{n}+\sum_{i=0}^{r} \delta q_{i} v_{n-h_{i}}+(1-\delta)\left(\Pi_{i=0}^{r} \beta_{i}\right)\left(v_{n-h_{i}}^{\alpha_{i}}\right)+\Upsilon, n \in \mathbb{Z}^{0} .
\end{aligned}
$$

Therefore, by Theorem 2.7, we obtain

$$
\left|x_{n}\right| \leq v_{n} \leq\left(\max _{-h_{r} \leq i \leq 0}\left\{v_{i}\right\}\right) \lambda_{0}^{n}+\Lambda=\left(\max _{-h_{r} \leq i \leq 0}\left\{\left|x_{i}\right|\right\}\right) \lambda_{0}^{n}+\Lambda, n \in \mathbb{Z}^{0},
$$

where $\Lambda=\left(p-\delta \sum_{i=0}^{r} q_{i}-(1-\delta) \Pi_{i=0}^{r} \beta_{i}\right)^{-1} \Upsilon$, and $\lambda_{0}$ is chosen as in Theorem 2.7. This completes the proof of the theorem.

Remark 3.6. When we take $\delta=0$. By Theorem 3.5 we have the following result.

Theorem 3.7. Assume that $0<p \leq 1$. Let $\Upsilon \in \mathbb{R}_{0}^{+}, h_{i} \in \mathbb{Z}^{0}, i=0, \ldots, r-1$; $h_{r} \in \mathbb{Z}^{+}$, where $0=h_{0}<h_{1}<\cdots<h_{r}$. Let $\alpha_{i}, \beta_{i} \in \mathbb{R}^{+}, \sum_{i=0}^{r} \alpha_{i}=1$ and $\Pi_{i=0}^{r} \beta_{i}<p \leq 1$. If

$$
\left|f\left(n, x_{n}, x_{n-h_{1}}, \ldots, x_{n-h_{r}}\right)\right| \leq\left(\Pi_{i=0}^{r} \beta_{i}\right)\left|x_{n-h_{i}}\right|^{\alpha_{i}}+\Upsilon
$$

for all $\left(n, x_{n}, x_{n-h-1}, \ldots, x_{n-h_{r}}\right) \in \mathbb{Z}^{0} \times \mathbb{R}^{r+1}$, then there exists $\lambda_{0} \in(0,1)$ such that, for every solution $\left\{x_{n}\right\}$ of equation (3.1),

$$
\left|x_{n}\right| \leq\left(\max _{-h_{r} \leq i \leq 0}\left\{\left|x_{i}\right|\right\}\right) \lambda_{0}^{n}+\Lambda, n \in \mathbb{Z}^{0},
$$

where $\Lambda=\left(p-\prod_{i=0}^{r} \beta_{i}\right)^{-1} \Upsilon$, and $\lambda_{0}$ is chosen as in Theorem 2.10. As a consequence, $S=\{\phi \in \mathbb{R}|| \phi \mid \leq \Lambda\}$ is a positive attracting set of (3.1).

Remark 3.8. When we take $\Upsilon=0$. By Theorem 3.5 we have the following result.

Theorem 3.9 ([1, Theorem 3.2]). Assume that $0<p \leq 1$. Let $q_{i} \in \mathbb{R}_{0}^{+}$, $h_{i} \in \mathbb{Z}^{0}, i=0, \ldots, r-1 ; a_{r}, q_{r} \in \mathbb{R}^{+}, h_{r} \in \mathbb{Z}^{+}$, where $0=h_{0}<h_{1}<\cdots<h_{r}$. Let $\alpha_{i}, \beta_{i} \in \mathbb{R}^{+}, \sum_{i=0}^{r} \alpha_{i}=1$ and $\left[(1-\delta) \Pi_{i=0}^{r} \beta_{i}+\delta \sum_{i=0}^{r} q_{i}\right]<p \leq 1$, where $0 \leq \delta \leq 1$ is a constant. If

$$
\left|f\left(n, x_{n}, x_{n-h_{1}}, \ldots, x_{n-h_{r}}\right)\right| \leq \sum_{i=0}^{r} \delta q_{i}\left|x_{n-h_{i}}\right|+(1-\delta)\left(\Pi_{i=0}^{r} \beta_{i}\right)\left|x_{n-h_{i}}\right|^{\alpha_{i}}
$$

for all $\left(n, x_{n}, x_{n-h-1}, \ldots, x_{n-h_{r}}\right) \in \mathbb{Z}^{0} \times \mathbb{R}^{r+1}$, then there exists $\lambda_{0} \in(0,1)$ such that, for every solution $\left\{x_{n}\right\}$ of equation (3.1),

$$
\left|x_{n}\right| \leq\left(\max _{-h_{r} \leq i \leq 0}\left\{\left|x_{i}\right|\right\}\right) \lambda_{0}^{n}, n \in \mathbb{Z}^{0},
$$


$\lambda_{0}$ is chosen as in Theorem 2.7. As a consequence, the trivial solution of the equation (3.1) is globally asymptotically stable.

\section{References}

[1] R. P. Agarwal, Y. H. Kim, and S. K. Sen, New discrete Halanay inequalities: stability of difference equations, Commun. Appl. Anal. 12 (2008), no. 1, 83-90.

[2] R. P. Agarwal and P. J. Y. Wong, Advanced Topics in Difference Equations, in: Mathematics and its Applications, vol. 404, Kluwer, Dordrecht, 1997.

[3] C. T. H. Baker, Development and application of Halanay-type theory: Evolutionary differential and difference equations with time lag, J. Comput. Appl. Math. 234 (2010), no. 9, 2663-2682.

[4] E. Beckenbach and R. Bellman, Inequalities, Springer-Verlag, New York, 1961.

[5] L. Berezansky, L. Idels, and L. Troib, Global dynamics of one class of nonlinear nonautonomous systems with time-varying delays, Nonlinear Anal. 74 (2011), no. 18, 74997512.

[6] J. D. Cao and J. Wang, Absolute exponential stability of recurrent neural networks with Lipschitz-continuous activation functions and time delays, Neural Networks 17 (2004), 379-390.

[7] E. Fridman and A. Blighovsky, Robust sampled-data control of a class of semilinear parabolic systems, Automatica J. IFAC 48 (2012), no. 5, 826-836.

[8] K. Gopalsamy, Stability and Oscillations in Delay Differential Equations of Population Dynamics, Kluwer Academic, Dordrecht, 1992.

[9] A. Halanay, Differential Equations: Stability, Oscillations, Time Lags, Academic Press, New York, 1966.

[10] E. Liz and J. B. Ferreiro, A Note on the global stability of generalized difference equations, Appl. Math. Lett. 15 (2002), no. 6, 655-659.

[11] E. Liz and S. Trofimchuk, Existence and stability of almost periodic solutions for quasilinear delay systems and Halanay inequality, J. Math. Anal. Appl. 248 (2000), no. 2, 625-644.

[12] S. Mohamad, Global exponential stability in continuous-time and discrete-time delayed bidirectional neural networks, Phys. D 159 (2001), no. 3-4, 233-251

[13] S. Mohamad, K. Gopalsamy, and H. Akca, Exponential stability of artificial neural networks with distributed delays and large impulses, Nonlinear Anal. Real World Appl. 9 (2008), no. 3, 872-888.

[14] P. Niamsup, Stability of time-varying switched systems with time-varying delay, Nonlinear Anal. Hybrid Syst. 3 (2009), no. 4, 631-639.

[15] H. J. Tian, The exponential asymptotic stability of singularly perturbed delay differential equations with a bounded lag, J. Math. Anal. Appl. 270 (2002), no. 1, 143-149.

[16] S. Udpin and P. Niamsup, New discrete type inequalities and global stability of nonlinear difference equations, Appl. Math. Lett. 22 (2009), no. 6, 856-859.

[17] L. P. Wen, Y. X. Yu, and W. S. Wang, Generalized Halanay inequalities for dissipativity of Volterra functional differential equations, J. Math. Anal. Appl. 347 (2008), no. 1, 169-178.

[18] D. Y. Xu and X. H. Wang, A new nonlinear integro-differential inequality and its application, Appl. Math. Lett. 22 (2009), no. 11, 1721-1726.

[19] D. Y. Xu and L. G. Xu, New results for studying a certain class of nonlinear delay differential systems, IEEE Trans. Automat. Control 55 (2010), no. 7, 1641-1645.

[20] D. Y. Xu and Z. C. Yang, Impulsive delay differential inequality and stability of neural networks, J. Math. Anal. Appl. 305 (2005), no. 1, 107-120.

[21] L. G. Xu, Exponential P-stability of singularly perturbed impulsive stochastic delay differential systems, Int. J. Control, Autom. 9 (2011), 966-972. 
[22] L. G. Xu and D. H. He, Mean square exponential stability analysis of impulsive stochastic switched systems with mixed delays, Comput. Math. Appl. 62 (2011), no. 1, 109-117.

[23] L. G. Xu and D. Y. Xu, Exponential stability of nonlinear impulsive neutral integrodifferential equations, Nonlinear Anal. 69 (2008), no. 9, 2910-2923.

[24] Z. G. Yang and D. Y. Xu, Mean square exponential stability of impulsive stochastic difference equations, Appl. Math. Lett. 20 (2007), no. 8, 938-945.

[25] H. Y. Zhao, L. Sun, and G. L. Wang, Periodic oscillation of discrete-time bidirectional associative memory neural networks, Neurocomputing 70 (2007), 2924-2930.

Department of Applied Mathematics

Zhejiang University of TeChNology

Hangzhou, 310023, P. R. China

E-mail address: xlg132@126.com 